ANNALES

POLONICI MATHEMATICI

$84.3(2004)$

\title{
Hyperbolically 1-convex functions
}

\author{
by William Ma (Williamsport, PA), David Minda (Cincinnati, OH) \\ and Diego Mejia (Medellín)
}

\begin{abstract}
There are two reasonable analogs of Euclidean convexity in hyperbolic geometry on the unit disk $\mathbb{D}$. One is hyperbolic convexity and the other is hyperbolic 1-convexity. Associated with each type of convexity is the family of univalent holomorphic maps of $\mathbb{D}$ onto subregions of the unit disk that are hyperbolically convex or hyperbolically 1-convex. The class of hyperbolically convex functions has been the subject of a number of investigations, while the family of hyperbolically 1-convex functions has received less attention. This paper is a contribution to the study of hyperbolically 1-convex functions. A main result is that a holomorphic univalent function $f$ defined on $\mathbb{D}$ with $f(\mathbb{D}) \subseteq \mathbb{D}$ is hyperbolically 1-convex if and only if $f /(1-w f)$ is a Euclidean convex function for each $w \in \overline{\mathbb{D}}$. This characterization gives rise to two-variable characterizations of hyperbolically 1-convex functions. These two-variable characterizations yield a number of sharp results for hyperbolically 1-convex functions. In addition, we derive sharp two-point distortion theorems for hyperbolically 1-convex functions.
\end{abstract}

1. Introduction. There are two natural analogs of Euclidean convexity in hyperbolic geometry. One of the two has received more attention than the other. First, a subregion $\Omega$ of the unit disk $\mathbb{D}=\{z:|z|<1\}$ is called hyperbolically convex (relative to hyperbolic geometry on $\mathbb{D}$ ) if for all points $A, B \in \Omega$ the arc of the hyperbolic geodesic in $\mathbb{D}$ connecting $A$ and $B$ (the arc of circle joining $A$ and $B$ that is orthogonal to the unit circle) lies in $\Omega$. A holomorphic and univalent function $f$ on $\mathbb{D}$ is called hyperbolically convex if its image $f(\mathbb{D})$ is a hyperbolically convex subset of $\mathbb{D}$. Hyperbolically convex functions have been studied in [4], [5], [7]-[9] and [11]. There is another notion of convexity in hyperbolic geometry that can be viewed as an analog of Euclidean convexity. A subregion $\Omega$ of $\mathbb{D}$ is called hyperbolically 1 -convex (relative to hyperbolic geometry on $\mathbb{D}$ ) if for any pair of points $A, B \in \Omega$ both of the two shorter circular arcs of constant hyperbolic curvature 1 in $\mathbb{D}$ connecting $A$ and $B$ lie in $\Omega$. Arcs of constant hyperbolic curvature 1 are

2000 Mathematics Subject Classification: Primary 30C45, 30C50.

Key words and phrases: hyperbolic 1-convexity, two-variable characterizations, order of convexity and starlikeness, two-point distortion theorems. 
arcs of horocycles; that is, circles in $\mathbb{D}$ that are tangent to $\partial \mathbb{D}$. Given distinct points $A$ and $B$ in $\mathbb{D}$ there are precisely two circles through $A$ and $B$ that are tangent to the unit circle. A holomorphic and univalent function $f$ on $\mathbb{D}$ is called hyperbolically 1 -convex if its image $f(\mathbb{D})$ is a hyperbolically 1 convex subset of $\mathbb{D}$. Hyperbolically 1-convex functions have been considered in [8] and [9]. In these references the functions are called hyperbolically 2 -convex due to a different normalization of the hyperbolic metric on $\mathbb{D}$. As observed in [9], hyperbolic 1-convexity in hyperbolic geometry often plays a role in hyperbolic geometry analogous to the roles of Euclidean convexity in Euclidean geometry and spherical convexity in spherical geometry, while hyperbolic convexity sometimes presents intriguing differences.

Throughout this paper, we let $\mathcal{B}$ be the family of all holomorphic functions $f$ in $\mathbb{D}$ with $f(\mathbb{D}) \subseteq \mathbb{D}$. We also denote by $K_{h}^{1}$ the family of all hyperbolically 1-convex functions in $\mathbb{D}$. Finally, $K_{h}^{1}(\alpha)$ is the subclass of normalized $\left(f(0)=0\right.$ and $\left.f^{\prime}(0)=\alpha \in(0,1)\right)$ functions in $K_{h}^{1}$.

Known one-variable characterizations for $K_{h}^{1}$ usually contain nonholomorphic terms that make such characterizations difficult to use. For example, a locally univalent function $f \in \mathcal{B}$ is in $K_{h}^{1}$ if and only if either of the following inequalities holds [8]:

$$
\begin{gathered}
\left|\left(1-|z|^{2}\right) \frac{f^{\prime \prime}(z)}{f^{\prime}(z)}-2 \bar{z}+\frac{2\left(1-|z|^{2}\right) \overline{f(z)} f^{\prime}(z)}{1-|f(z)|^{2}}\right| \\
\leq 2\left(1-\frac{\left(1-|z|^{2}\right)\left|f^{\prime}(z)\right|}{1-|f(z)|^{2}}\right), \\
\operatorname{Re}\left\{1+\frac{z f^{\prime \prime}(z)}{f^{\prime}(z)}+\frac{2 \overline{f(z)} z f^{\prime}(z)}{1-|f(z)|^{2}}\right\} \geq \frac{2\left|z f^{\prime}(z)\right|}{1-|f(z)|^{2}} .
\end{gathered}
$$

Our principal goal is to extend an idea [8] used in establishing distortion theorems for $K_{h}^{1}$. Precisely, we give a transformation that converts functions of $K_{h}^{1}$ into Euclidean convex functions; this transformation characterizes functions in $K_{h}^{1}$. The transformation gives a two-variable characterization for functions in $K_{h}^{1}$ that is holomorphic in each of the two variables. By using this two-variable characterization, we obtain a number of results for $K_{h}^{1}$ including connections with starlike functions and best possible lower bounds on $\operatorname{Re}\left\{f^{\prime \prime}(0) f(z)\right\}, \operatorname{Re}\left\{z f^{\prime}(z) / f(z)\right\}$ and $\operatorname{Re}\left\{1+z f^{\prime \prime}(z) / f^{\prime}(z)\right\}$. We also give sharp upper and lower estimates on the "hyperbolic derivative" $\left(1-|z|^{2}\right)\left|f^{\prime}(z)\right| /\left(1-|f(z)|^{2}\right)$. Finally, we derive sharp two-point distortion theorems for $K_{h}^{1}$.

2. Preliminaries. Let $\Omega$ be a region in $\mathbb{D}$. The hyperbolic metric on $\Omega$ is denoted by $\lambda_{\Omega}(w)|d w|$ and is normalized to have curvature 


$$
-\frac{\Delta \log \lambda_{\Omega}(w)}{\lambda_{\Omega}^{2}(w)}=-1
$$

where $w=u+i v$ and

$$
\Delta=\frac{\partial^{2}}{\partial u^{2}}+\frac{\partial^{2}}{\partial v^{2}}=4 \frac{\partial^{2}}{\partial w \partial \bar{w}}
$$

denotes the usual Laplacian. The hyperbolic metric on the unit disk $\mathbb{D}$ is $\lambda_{\mathbb{D}}(z)|d z|=2|d z| /\left(1-|z|^{2}\right)$ and the associated hyperbolic distance on $\mathbb{D}$ is

$$
d_{\mathbb{D}}(a, b)=2 \tanh ^{-1}\left|\frac{a-b}{1-\bar{b} a}\right| .
$$

If $f: \mathbb{D} \rightarrow \Omega$ is any holomorphic universal covering projection, then the density $\lambda_{\Omega}$ of the hyperbolic metric is determined from $\lambda_{\Omega}(f(z))\left|f^{\prime}(z)\right|=$ $2 /\left(1-|z|^{2}\right)$.

It is convenient to introduce certain invariant differential operators defined for functions in $\mathcal{B}$. If $f \in \mathcal{B}$, set

$$
\begin{aligned}
D_{h 1} f(z) & =\frac{\left(1-|z|^{2}\right) f^{\prime}(z)}{1-|f(z)|^{2}}, \\
D_{h 2} f(z) & =\frac{\left(1-|z|^{2}\right)^{2} f^{\prime \prime}(z)}{1-|f(z)|^{2}}-\frac{2 \bar{z}\left(1-|z|^{2}\right) f^{\prime}(z)}{1-|f(z)|^{2}}+\frac{2\left(1-|z|^{2}\right)^{2} \overline{f(z)} f^{\prime}(z)^{2}}{\left(1-|f(z)|^{2}\right)^{2}}, \\
D_{h 3} f(z) & =\frac{\left(1-|z|^{2}\right)^{3} f^{\prime \prime \prime}(z)}{1-|f(z)|^{2}}+\frac{6\left(1-|z|^{2}\right)^{3} \overline{f(z)} f^{\prime}(z) f^{\prime \prime}(z)}{1-|f(z)|^{2}} \\
& -\frac{6 \bar{z}\left(1-|z|^{2}\right)^{2} f^{\prime \prime}(z)}{1-|f(z)|^{2}}+\frac{6 \bar{z}^{2}\left(1-|z|^{2}\right) f^{\prime}(z)}{1-|f(z)|^{2}} \\
& -\frac{12 \bar{z}\left(1-|z|^{2}\right)^{2} \overline{f(z)} f^{\prime}(z)^{2}}{\left(1-|f(z)|^{2}\right)^{2}}+\frac{6\left(1-|z|^{2}\right)^{3} \overline{f(z)} f^{\prime}(z)^{3}}{\left(1-|f(z)|^{2}\right)^{3}} .
\end{aligned}
$$

These differential operators satisfy the important invariance property that $\left|D_{h j}(S \circ f \circ T)\right|=\left|D_{h j} f\right| \circ T, j=1,2,3$, whenever $S$ and $T$ are conformal automorphisms of $\mathbb{D}$. In particular, $D_{h j} f(0)=f^{(j)}(0), j=1,2,3$, if $f(0)=0$. More information about these operators can be found in [4]. For locally univalent functions in $\mathcal{B}$, the following combinations of these operators are used frequently:

$$
Q_{f}(z)=\frac{D_{h 2} f(z)}{D_{h 1} f(z)}=\left(1-|z|^{2}\right) \frac{f^{\prime \prime}(z)}{f^{\prime}(z)}-2 \bar{z}+\frac{2\left(1-|z|^{2}\right) \overline{f(z)} f^{\prime}(z)}{1-|f(z)|^{2}}
$$

and

$$
\frac{D_{h 3} f(z)}{D_{h 1} f(z)}-\frac{3}{2}\left(\frac{D_{h 2} f(z)}{D_{h 1} f(z)}\right)^{2}=\left(1-|z|^{2}\right)^{2} S_{f}(z)
$$


where

$$
S_{f}(z)=\frac{f^{\prime \prime \prime}(z)}{f^{\prime}(z)}-\frac{3}{2}\left(\frac{f^{\prime \prime}(z)}{f^{\prime}(z)}\right)^{2}
$$

is the Schwarzian derivative of $f$.

For $f(z)=\alpha z+a_{2} z^{2}+\cdots \in K_{h}^{1}(\alpha)$, we have $\left|a_{2}\right| \leq \alpha(1-\alpha)$ ([8]). This inequality is sharp. Equality holds for

$$
k_{\alpha}(z)=\frac{\alpha z}{1-(1-\alpha) z}
$$

and its rotations; these functions map $\mathbb{D}$ onto a horodisk. The invariant form of the coefficient inequality $\left|a_{2}\right| \leq \alpha(1-\alpha)$ is

$$
\left|Q_{f}(z)\right| \leq 2\left(1-\left|D_{h 1} f(z)\right|\right)
$$

for all $z \in \mathbb{D}$. For $k_{\alpha}$ equality holds in $(2.2)$ on the interval $(-1,1)$. Inequality (2.2) characterizes functions in $K_{h}^{1}$; it is (1.1) in different notation. In [8], the authors also proved the following growth theorem for $K_{h}^{1}(\alpha)$ :

$$
-k_{\alpha}(-|z|) \leq|f(z)| \leq k_{\alpha}(|z|) \text {. }
$$

3. Transforming hyperbolically 1-convex functions into Euclidean convex functions. Clearly, a hyperbolically 1-convex region $\Omega \subseteq \mathbb{D}$ is Euclidean convex. We use this observation to derive a characterization of functions of $K_{h}^{1}$ in terms of a transformation. A similar idea was used by Mejia and Pommerenke [10] in their investigation of spherically convex functions.

Theorem 3.1. For $f \in \mathcal{B}, f \in K_{h}^{1}$ if and only if $f /(1-w f)$ is Euclidean convex for every $w \in \overline{\mathbb{D}}$.

Proof. Suppose $f \in K_{h}^{1}$. Then for each $w \in \mathbb{D}$,

$$
f_{w}=\frac{f-\bar{w}}{1-w f} \in K_{h}^{1},
$$

and so $f_{w}$ is Euclidean convex. As

$$
\frac{f_{w}+\bar{w}}{1-|w|^{2}}=\frac{f}{1-w f},
$$

we deduce that $f /(1-w f)$ is Euclidean convex for any $w \in \mathbb{D}$. A limit argument implies that $f /(1-w f)$ is Euclidean convex for every $w \in \overline{\mathbb{D}}$.

Conversely, we show $f \in K_{h}^{1}$ if $f /(1-w f)$ is Euclidean convex for every $w \in \overline{\mathbb{D}}$. First, we prove that for any $a \in \mathbb{D}, f_{a}=(f-\bar{a}) /(1-a f)$ also satisfies the same assumption, that is, $f_{a} /\left(1-w f_{a}\right)$ is Euclidean convex for every $w \in \overline{\mathbb{D}}$. This follows from

$$
\frac{f_{a}}{1-w f_{a}}=\frac{f-\bar{a}}{1+\bar{a} w-(a+w) f}=\frac{\left(1-\bar{a} \frac{a+w}{1+\bar{a} w}\right) f}{(1+\bar{a} w)\left(1-\frac{a+w}{1+\bar{a} w} f\right)}-\frac{\bar{a}}{1+\bar{a} w}
$$


and $\frac{a+w}{1+\bar{a} w} \in \overline{\mathbb{D}}$. Thus, we can assume $f(0)=0$. By assumption,

$$
\frac{f(z)}{f^{\prime}(0)\left(1-e^{-i \varphi} f(z)\right)}=z+\left(\frac{1}{2} \frac{f^{\prime \prime}(0)}{f^{\prime}(0)}+e^{-i \varphi} f^{\prime}(0)\right) z^{2}+\cdots
$$

is a normalized Euclidean convex function for each $\varphi \in \mathbb{R}$, so

$$
\left|f^{\prime \prime}(0) /\left(2 f^{\prime}(0)\right)+e^{-i \varphi} f^{\prime}(0)\right| \leq 1 .
$$

An appropriate choice of $\varphi$ gives

$$
\left|f^{\prime \prime}(0) / f^{\prime}(0)\right| \leq 2\left(1-\left|f^{\prime}(0)\right|\right) .
$$

If we apply this inequality to

$$
F_{\zeta}(z)=\frac{f\left(\frac{z+\zeta}{1+\bar{\zeta} z}\right)-f(\zeta)}{1-\overline{f(\zeta)} f\left(\frac{z+\zeta}{1+\bar{\zeta} z}\right)}
$$

which satisfies our assumption, we obtain, for any $\zeta \in \mathbb{D}$,

$$
\left|Q_{f}(\zeta)\right|=\left|\frac{D_{h 2} f(\zeta)}{D_{h 1} f(\zeta)}\right|=\left|\frac{F_{\zeta}^{\prime \prime}(0)}{F_{\zeta}^{\prime}(0)}\right| \leq 2\left(1-\left|F_{\zeta}^{\prime}(0)\right|\right)=2\left(1-\left|D_{h 1} f(\zeta)\right|\right) .
$$

Therefore, $f \in K_{h}^{1}$.

In [8], the authors showed $f /\left(1-e^{-i \varphi} f\right)$ is Euclidean convex for all $\varphi \in \mathbb{R}$ when $f \in K_{h}^{1}$. Theorem 3.1 is a stronger result and characterizes $K_{h}^{1}$. Theorem 3.1 is analogous to a result on spherically convex functions due to Mejia and Pommerenke [10]; however, Theorem 3.1 does not require the normalization $f(0)=0$ as does the result in [10].

4. Two-variable characterizations. Theorem 3.1 yields two-variable characterizations for $K_{h}^{1}$ that are holomorphic in each variable. Recall that a holomorphic function $g$ in $\mathbb{D}$ is Euclidean convex if and only if (see [13] and [14])

$$
\operatorname{Re}\left\{\frac{2 z g^{\prime}(z)}{g(z)-g(\zeta)}-\frac{z+\zeta}{z-\zeta}\right\}>0 \quad(z, \zeta \in \mathbb{D}) .
$$

Theorem 4.1. Suppose $f \in \mathcal{B}$. Then $f \in K_{h}^{1}$ if and only if

$$
\operatorname{Re}\left\{\frac{2 z f^{\prime}(z)}{f(z)-f(\zeta)}-\frac{z+\zeta}{z-\zeta}+\frac{2 w z f^{\prime}(z)}{1-w f(z)}\right\}>0 \quad(z, \zeta \in \mathbb{D})
$$

holds for all $w \in \overline{\mathbb{D}}$.

Proof. By using Theorem 3.1 and (4.1), we have

$$
\operatorname{Re}\left\{\frac{2 z f^{\prime}(z)}{f(z)-f(\zeta)} \frac{1-w f(\zeta)}{1-w f(z)}-\frac{z+\zeta}{z-\zeta}\right\}>0 .
$$

This is equivalent to (4.2). 
Theorem 4.1 with the special choice $w=\overline{f(z)}$ characterizes hyperbolically convex functions (see [11] and [7]). We now give a symmetric twovariable characterization.

Corollary 4.2. Suppose $f \in \mathcal{B}$. Then $f \in K_{h}^{1}$ if and only if

$$
\operatorname{Re}\left\{\frac{z f^{\prime}(z)-\zeta f^{\prime}(\zeta)}{f(z)-f(\zeta)}+\frac{w z f^{\prime}(z)}{1-w f(z)}+\frac{\omega \zeta f^{\prime}(\zeta)}{1-\omega f(\zeta)}\right\}>0 \quad(z, \zeta \in \mathbb{D})
$$

for all $w, \omega \in \overline{\mathbb{D}}$.

Proof. If $f \in K_{h}^{1}$, then (4.2) holds. By interchanging $z$ and $\zeta$, and replacing $w$ by $\omega$, we obtain

$$
\operatorname{Re}\left\{-\frac{2 \zeta f^{\prime}(\zeta)}{f(z)-f(\zeta)}+\frac{z+\zeta}{z-\zeta}+\frac{2 \omega \zeta f^{\prime}(\zeta)}{1-\omega f(\zeta)}\right\}>0
$$

By adding this inequality to (4.2) and then dividing by 2 , we get (4.3).

Next, assume (4.3) holds. In particular, when $\zeta=z$ and $\omega=w$, (4.3) becomes

$$
\operatorname{Re}\left\{1+\frac{z f^{\prime \prime}(z)}{f^{\prime}(z)}+\frac{2 w z f^{\prime}(z)}{1-w f(z)}\right\} \geq 0 .
$$

Let

$$
w=\frac{\overline{f(z)}-\frac{\left|z f^{\prime}(z)\right|}{z f^{\prime}(z)}}{1-f(z) \frac{\left|z f^{\prime}(z)\right|}{z f^{\prime}(z)}}
$$

and note $|w|=1$ since $\frac{\left|z f^{\prime}(z)\right|}{z f^{\prime}(z)} \in \partial \mathbb{D}$ for $z \neq 0$. Direct calculation shows

$$
\frac{w z f^{\prime}(z)}{1-w f(z)}=\frac{\overline{f(z)} z f^{\prime}(z)}{1-|f(z)|^{2}}-\frac{\left|z f^{\prime}(z)\right|}{1-|f(z)|^{2}}
$$

Therefore, for $z \in \mathbb{D} \backslash\{0\}$,

$$
\operatorname{Re}\left\{1+\frac{z f^{\prime \prime}(z)}{f^{\prime}(z)}+\frac{2 \overline{f(z)} z f^{\prime}(z)}{1-|f(z)|^{2}}-\frac{2\left|z f^{\prime}(z)\right|}{1-|f(z)|^{2}}\right\} \geq 0
$$

and the inequality trivially holds for $z=0$. This is equivalent to (1.2), which characterizes $K_{h}^{1}$.

5. Applications. Recall that a holomorphic and univalent function $g$ normalized by $g(0)=g^{\prime}(0)-1=0$ is called starlike of order $\beta$ if $\operatorname{Re}\left\{z g^{\prime}(z) / g(z)\right\}>\beta$ in $\mathbb{D}$. if

Theorem 5.1. Suppose $f \in \mathcal{B}$ and $f(0)=0$. Then $f \in K_{h}^{1}$ if and only

$$
F_{\zeta, w}(z)=\frac{z \zeta}{f(\zeta)} \frac{f(z)-f(\zeta)}{(z-\zeta)(1-w f(z))}
$$

is starlike of order $1 / 2$ for each $\zeta \in \mathbb{D}$ and each $w \in \overline{\mathbb{D}}$. 
Proof. Direct calculations give

$$
\frac{2 z F_{\zeta, w}^{\prime}(z)}{F_{\zeta, w}(z)}-1=\frac{2 z f^{\prime}(z)}{f(z)-f(\zeta)}-\frac{z+\zeta}{z-\zeta}+\frac{2 w z f^{\prime}(z)}{1-w f(z)} .
$$

Thus, Theorem 5.1 follows from Theorem 4.1.

For the special value $w=\overline{f(\zeta)}$, the analogous result characterizes hyperbolically convex functions [6].

Corollary 5.2. If $f \in K_{h}^{1}$ with $f(0)=0$, then

$$
\operatorname{Re}\left\{\frac{\zeta}{f(\zeta)} \frac{f(z)-f(\zeta)}{(z-\zeta)(1-w f(z))}\right\}>\frac{1}{2}
$$

for all $\zeta \in \mathbb{D}$ and all $w \in \overline{\mathbb{D}}$. In particular, if $f \in K_{h}^{1}(\alpha)$, then for $z \in \mathbb{D}$,

$$
\operatorname{Re}\left\{z f^{\prime}(z) / f(z)\right\}>1 /(2-\alpha) .
$$

Inequality (5.1) is best possible for each $\alpha \in(0,1)$.

Proof. It is known (see [12]) that $\operatorname{Re}\{F(z) / z\}>1 / 2$ if $F$ is starlike of order $1 / 2$. Then Theorem 5.1 implies the first inequality. For $\zeta=z$, the first inequality becomes

$$
\operatorname{Re}\left\{\frac{z f^{\prime}(z)}{f(z)(1-w f(z))}\right\}>\frac{1}{2} .
$$

The choice $w=-\overline{f(z)} /|f(z)|$ produces

$$
\operatorname{Re}\left\{z f^{\prime}(z) / f(z)\right\}>(1+|f(z)|) / 2 .
$$

From the lower bound in (2.3),

$$
\lim _{|z| \rightarrow 1} \frac{1}{2}(1+|f(z)|) \geq \frac{1}{2}\left(1+\frac{\alpha}{2-\alpha}\right)=\frac{1}{2-\alpha} .
$$

Because $\operatorname{Re}\left\{z f^{\prime}(z) / f(z)\right\}$ is harmonic in $\mathbb{D}$, we obtain (5.1).

For the function $k_{\alpha}, \inf \left\{\operatorname{Re}\left\{z k_{\alpha}^{\prime}(z) / k_{\alpha}(z)\right\}: z \in \mathbb{D}\right\}=1 /(2-\alpha)$, so (5.1) is best possible for each $\alpha \in(0,1)$.

Corollary 5.3. If $f \in K_{h}^{1}$ with $f(0)=0$, then for every $\zeta \in \mathbb{D}$ and $w \in \overline{\mathbb{D}}$,

$$
\frac{F_{\zeta, w}^{2}(z)}{z}=\frac{z \zeta^{2}}{f(\zeta)^{2}} \frac{(f(z)-f(\zeta))^{2}}{(z-\zeta)^{2}(1-w f(z))^{2}}
$$

is starlike in $\mathbb{D}$.

Now we derive the sharp order of Euclidean convexity for functions in $K_{h}^{1}(\alpha)$. 
Theorem 5.4. If $f \in K_{h}^{1}(\alpha)$, then for $z \in \mathbb{D}$,

$$
\operatorname{Re}\left\{1+\frac{z f^{\prime \prime}(z)}{f^{\prime}(z)}\right\}>\frac{\alpha}{2-\alpha} .
$$

This result is best possible for each $\alpha \in(0,1)$.

Proof. For $\zeta=z,(4.2)$ becomes

$$
\operatorname{Re}\left\{1+\frac{z f^{\prime \prime}(z)}{f^{\prime}(z)}+\frac{2 w z f^{\prime}(z)}{1-w f(z)}\right\}>0 .
$$

The choice $w=-\overline{f(z)} /|f(z)|$ gives

$$
\operatorname{Re}\left\{1+\frac{z f^{\prime \prime}(z)}{f^{\prime}(z)}\right\}>2 \frac{|f(z)|}{1+|f(z)|} \operatorname{Re}\left\{\frac{z f^{\prime}(z)}{f(z)}\right\} .
$$

From (2.3),

$$
\frac{|f(z)|}{1+|f(z)|} \geq \frac{\alpha|z|}{(1+(1-\alpha)|z|)\left(1+\frac{\alpha|z|}{1+(1-\alpha)|z|}\right)}=\frac{\alpha|z|}{1+|z|} .
$$

This together with Corollary 5.2 yields

$$
\operatorname{Re}\left\{1+\frac{z f^{\prime \prime}(z)}{f^{\prime}(z)}\right\}>\frac{2}{2-\alpha} \frac{\alpha|z|}{1+|z|} .
$$

The right-hand side approaches $\frac{\alpha}{2-\alpha}$ as $|z| \rightarrow 1$. As $\operatorname{Re}\left\{1+z f^{\prime \prime}(z) / f^{\prime}(z)\right\}$ is harmonic in $\mathbb{D}$, we get (5.2). For $k_{\alpha}$,

$$
\inf \left\{\operatorname{Re}\left\{1+\frac{z k_{\alpha}^{\prime \prime}(z)}{k_{\alpha}^{\prime}(z)}\right\}: z \in \mathbb{D}\right\}=\frac{\alpha}{2-\alpha},
$$

so (5.2) is best possible for each $\alpha$.

For locally univalent holomorphic functions, the order of starlikeness is clearly at least the same as the order of convexity. The first part of our next result provides a comparison in the other direction for $f \in K_{h}^{1}(\alpha)$, which will be used to improve the known order of growth of $\left|f^{\prime}(z)\right|$ when $\alpha>1 / 2$. In [8], we obtained a sharp upper bound on $\left|f^{\prime}(z)\right|$ for $f \in K_{h}^{1}(\alpha)$ only when $|z| \leq 2 /(1+\sqrt{1+4 \alpha})$, and also showed $\left|f^{\prime}(z)\right|=O(1 /(1-|z|))$ as $|z| \rightarrow 1$. For $|z|$ close to 1 , the sharp upper bound on $\left|f^{\prime}(z)\right|$ remains unknown.

Theorem 5.5. If $f \in K_{h}^{1}(\alpha)$, then for $z \in \mathbb{D}$,

$$
\operatorname{Re}\left\{1+\frac{z f^{\prime \prime}(z)}{f^{\prime}(z)}\right\}>\alpha \operatorname{Re}\left\{\frac{z f^{\prime}(z)}{f(z)}\right\}
$$

and

$$
\left|f^{\prime}(z)\right| \leq \frac{\alpha}{(1-|z|)^{2(1-\alpha)}}\left|\frac{f(z)}{\alpha z}\right|^{\alpha}<\frac{\alpha^{1-\alpha}}{(1-|z|)^{2(1-\alpha)}}
$$


Proof. From (5.3) and (5.4),

$$
\operatorname{Re}\left\{1+\frac{z f^{\prime \prime}(z)}{f^{\prime}(z)}\right\}>\frac{2 \alpha r}{1+r} \operatorname{Re}\left\{\frac{z f^{\prime}(z)}{f(z)}\right\}
$$

on $|z| \leq r$ for each fixed $r, 0<r<1$. By letting $r \rightarrow 1$ and noting the two sides cannot be identical when $\alpha<1$, (5.5) holds.

In order to derive the upper bound on $\left|f^{\prime}(z)\right|$, we define

$$
F(z)=\frac{z f^{\prime}(z)}{\alpha\left(\frac{f(z)}{\alpha z}\right)^{\alpha}}
$$

Then

$$
\frac{z F^{\prime}(z)}{F(z)}=1+\frac{z f^{\prime \prime}(z)}{f^{\prime}(z)}-\alpha \frac{z f^{\prime}(z)}{f(z)}+\alpha .
$$

This together with (5.5) implies that $F$ is starlike of order $\alpha$ and so (see $[3])|F(z)| \leq|z| /(1-|z|)^{2(1-\alpha)}$. This is equivalent to the second inequality in Theorem 5.5.

If $f$ is meromorphic and satisfies $\left(1-|z|^{2}\right)^{2}\left|S_{f}(z)\right| \leq 2$ in $\mathbb{D}$, then $f$ is called a Nehari function. Chuaqui and Osgood [1] proved that for normalized $\left(f(0)=f^{\prime}(0)-1=0\right)$ holomorphic Nehari functions, $a_{2} f(z) \neq-1$ in $\mathbb{D}$, where $a_{2}=f^{\prime \prime}(0) / 2$. For normalized Euclidean convex functions $f$, Fournier, Ma and Ruscheweyh [2] established the stronger result $\operatorname{Re}\left\{a_{2} f(z)\right\}>-1 / 2$, $z \in \mathbb{D}$. Related inequalities hold for hyperbolically convex functions [7].

TheOREM 5.6. If $f \in K_{h}^{1}(\alpha)$, then

$$
\operatorname{Re}\left\{a_{2} f(z)\right\}>-\alpha^{2} \frac{1-\alpha}{2-\alpha}
$$

for all $z \in \mathbb{D}$. The result is best possible for each $\alpha \in(0,1)$.

Proof. By Theorem 4.1, the function

$$
p_{\zeta, w}(z)=\frac{2 z f^{\prime}(z)}{f(z)-f(\zeta)}-\frac{z+\zeta}{z-\zeta}+\frac{2 w z f^{\prime}(z)}{1-w f(z)}
$$

is holomorphic and has positive real part in $\mathbb{D}$. Direct calculation yields

$$
\frac{1}{2} p_{\zeta, w}^{\prime}(0)=-\frac{\alpha}{f(\zeta)}+\frac{1}{\zeta}+\alpha w
$$

and

$$
\frac{1}{8}\left(p_{\zeta, w}^{\prime \prime}(0)-p_{\zeta, w}^{\prime}(0)^{2}\right)=(1-w f(\zeta))\left(-\frac{a_{2}}{f(\zeta)}-\frac{\alpha^{2}}{f(\zeta)^{2}}+\frac{\alpha}{\zeta f(\zeta)}\right) .
$$

From $\left|p_{\zeta, w}^{\prime \prime}(0)-p_{\zeta, w}^{\prime}(0)^{2}\right|+\left|p_{\zeta, w}^{\prime}(0)\right|^{2} \leq 4$ (see [5]), we obtain

$$
|1-w f(\zeta)|\left|a_{2} f(\zeta)+\alpha^{2}-\frac{\alpha f(\zeta)}{\zeta}\right|+\frac{1}{2}\left|\frac{f(\zeta)}{\zeta}-\alpha(1-w f(\zeta))\right|^{2} \leq \frac{1}{2}|f(\zeta)|^{2} .
$$


By choosing $w=-\overline{f(\zeta)} /|f(\zeta)|$, we find

$\left|a_{2} f(\zeta)+\alpha^{2}-\frac{\alpha f(\zeta)}{\zeta}\right| \leq \frac{1}{2(1+|f(\zeta)|)}\left(|f(\zeta)|^{2}-\left|\frac{f(\zeta)}{\zeta}-\alpha(1+|f(\zeta)|)\right|^{2}\right)$.

Therefore,

$$
\begin{aligned}
\operatorname{Re}\left\{a_{2} f(\zeta)\right\} \geq & -\alpha^{2}+\alpha \operatorname{Re}\{f(\zeta) / \zeta\} \\
& -\frac{1}{2(1+|f(\zeta)|)}\left(|f(\zeta)|^{2}-\left|\frac{f(\zeta)}{\zeta}-\alpha(1+|f(\zeta)|)\right|^{2}\right) \\
= & \frac{1-|\zeta|^{2}}{2(1+|f(\zeta)|)}\left|\frac{f(\zeta)}{\zeta}\right|^{2}-\frac{\alpha^{2}}{2}+\frac{\alpha^{2}}{2}|f(\zeta)| \\
> & -\frac{\alpha^{2}}{2}+\frac{\alpha^{2}}{2}|f(\zeta)| .
\end{aligned}
$$

If $a_{2}=0$, the desired inequality clearly holds. If $a_{2} \neq 0$, then $\operatorname{Re}\left\{a_{2} f(\zeta)\right\}$ is a non-constant harmonic function of $\zeta$. By using (2.3) and the fact that this lower bound tends to $\alpha /(2-\alpha)$ as $|\zeta| \rightarrow 1$, we obtain, for any $\zeta \in \mathbb{D}$,

$$
\operatorname{Re}\left\{a_{2} f(\zeta)\right\}>-\alpha^{2} \frac{1-\alpha}{2-\alpha}
$$

It is straightforward to check that the infimum of $\operatorname{Re}\left\{a_{2} k_{\alpha}(z)\right\}$ over $z \in \mathbb{D}$ is $-\alpha^{2}(1-\alpha) /(2-\alpha)$.

Theorem 5.7. Suppose $f \in \mathcal{B}$ is locally univalent. Then $f \in K_{h}^{1}$ if and only if

$$
\left(1-|z|^{2}\right)^{2}\left|S_{f}(z)\right|+\frac{1}{2}\left(\left|\frac{D_{h 2} f(z)}{D_{h 1} f(z)}\right|+2\left|D_{h 1} f(z)\right|\right)^{2} \leq 2
$$

for all $z \in \mathbb{D}$. The constant 2 is best possible.

Proof. First, assume $f \in K_{h}^{1}$. Because of the invariance of the quantities involved, it suffices to establish (5.6) in the special case that $z=0$ and $f(0)=0$. From Theorem 3.1, $g=f /(1-w f)$ satisfies Trimble's inequality $($ see $[15])$

$$
\left|S_{g}(0)\right|+\frac{1}{2}\left|g^{\prime \prime}(0) / g^{\prime}(0)\right|^{2} \leq 2 .
$$

Because $S_{g}(0)=S_{f}(0)$ and $g^{\prime \prime}(0) / g^{\prime}(0)=f^{\prime \prime}(0) / f^{\prime}(0)+2 w f^{\prime}(0)$, we obtain

$$
\left|S_{f}(0)\right|+\frac{1}{2}\left|f^{\prime \prime}(0) / f^{\prime}(0)+2 w f^{\prime}(0)\right|^{2} \leq 2 .
$$

By choosing $w$ with $|w|=1$ so that

$$
\left|f^{\prime \prime}(0) / f^{\prime}(0)+2 w f^{\prime}(0)\right|=\left|f^{\prime \prime}(0) / f^{\prime}(0)\right|+2\left|f^{\prime}(0)\right|,
$$

we get (5.6) when $z=0$ and $f(0)=0$. Conversely, (5.6) clearly implies (2.2), which characterizes $K_{h}^{1}$. 
Next, we show that the constant 2 is best possible. For any $a, 0<a<1$, it is straightforward to check that the function

$$
h_{a}(z)=a \frac{\left(\frac{1+z}{1-z}\right)^{4 \arctan a / \pi}-1}{\left(\frac{1+z}{1-z}\right)^{4 \arctan a / \pi}+1}
$$

maps $\mathbb{D}$ univalently onto the lens-shaped region that is the intersection of the two horodisks bounded by the two horocycles passing through $-a$ and $a$. Thus, $h_{a} \in K_{h}^{1}$. Direct calculations show that

$$
S_{h_{a}}(0)=2\left(1-16 \arctan ^{2} a / \pi^{2}\right),
$$

which tends to 2 as $a$ approaches 0 . This implies that the constant 2 is best possible.

Theorem 5.7 is the analog for $K_{h}^{1}$ of the invariant form of Trimble's inequality for Euclidean convex functions.

6. Sharp bounds on the hyperbolic derivative. In this section, we derive sharp upper and lower bounds on the hyperbolic derivative $\left|D_{h 1} f(z)\right|$ for functions in $K_{h}^{1}$.

Theorem 6.1. Suppose $f \in K_{h}^{1}$. Then for any $a, b \in \mathbb{D}$, we have

$$
\frac{\left|D_{h 1} f(a)\right| e^{-L}}{1-\left(1-e^{-L}\right)\left|D_{h 1} f(a)\right|} \leq\left|D_{h 1} f(b)\right| \leq \frac{\left|D_{h 1} f(a)\right| e^{L}}{1+\left(e^{L}-1\right)\left|D_{h 1} f(a)\right|},
$$

where $L=d_{\mathbb{D}}(a, b)$. In particular, if $f \in K_{h}^{1}(\alpha)$, then

$$
\frac{\alpha(1-|z|)}{1+(1-2 \alpha)|z|} \leq\left|D_{h 1} f(z)\right| \leq \frac{\alpha(1+|z|)}{1-(1-2 \alpha)|z|} .
$$

All of these bounds are sharp.

Proof. We need only consider those $f$ that are not conformal automorphisms of $\mathbb{D}$ since the inequalities clearly hold for conformal automorphisms of $\mathbb{D}$. For any pair of fixed $a, b \in \mathbb{D}$, let $\gamma: z=z(s), 0 \leq s \leq L=d_{\mathbb{D}}(a, b)$, be the hyperbolic geodesic connecting $a, b$ and parametrized by hyperbolic arc length. Then $z^{\prime}(s)=\frac{1}{2}\left(1-|z(s)|^{2}\right) e^{i \theta(s)}$, where $e^{i \theta(s)}$ is the Euclidean unit tangent of $\gamma$ at $z(s)$. From [4] we have

$$
\frac{\frac{d}{d s}\left|D_{h 1} f(z(s))\right|}{\left|D_{h 1} f(z(s))\right|}=\frac{1}{2} \operatorname{Re}\left\{Q_{f}(z(s)) e^{i \theta(s)}\right\},
$$

and applying (2.2) we find that

$$
\frac{\frac{d}{d s}\left|D_{h 1} f(z(s))\right|}{\left|D_{h 1} f(z(s))\right|\left(1-\left|D_{h 1} f(z(s))\right|\right)} \leq 1 .
$$


By integrating this inequality over $[0, L]$, we obtain

$$
\log \frac{\left|D_{h 1} f(b)\right|\left(1-\left|D_{h 1} f(a)\right|\right)}{\left(1-\left|D_{h 1} f(b)\right|\right)\left|D_{h 1} f(a)\right|} \leq L,
$$

or

$$
\frac{\left|D_{h 1} f(b)\right|}{1-\left|D_{h 1} f(b)\right|} \leq \frac{\left|D_{h 1} f(a)\right|}{1-\left|D_{h 1} f(a)\right|} e^{L} .
$$

This is equivalent to the upper bound in (6.1). The lower bound in (6.1) is obtained by interchanging $a$ and $b$ in the upper bound.

If $f \in K_{h}^{1}(\alpha)$, then $\left|D_{h 1} f(0)\right|=\alpha$. By setting $a=0$ and $b=z$ in (6.1), we find

$$
\frac{\alpha \frac{1-|z|}{1+|z|}}{1-\alpha+\alpha \frac{1-|z|}{1+|z|}} \leq\left|D_{h 1} f(z)\right| \leq \frac{\alpha \frac{1+|z|}{1-|z|}}{1-\alpha+\alpha \frac{1+|z|}{1-|z|}}
$$

since $e^{L}=e^{d_{\mathbb{D}}(0, z)}=(1+|z|) /(1-|z|)$. Simplifying both sides gives (6.2).

For $k_{\alpha}$, direction calculation gives

$$
\left|D_{h 1} k_{\alpha}(r)\right|=\frac{\alpha\left(1-r^{2}\right)}{(1-(1-\alpha) r)^{2}-\alpha^{2} r^{2}}=\frac{\alpha(1+r)}{1-(1-2 \alpha) r} .
$$

This shows that the upper bounds are sharp. The sharpness of the lower bounds follows from

$$
\left|D_{h 1} k_{\alpha}(-r)\right|=\frac{\alpha\left(1-r^{2}\right)}{(1+(1-\alpha) r)^{2}-\alpha^{2} r^{2}}=\frac{\alpha(1-r)}{1+(1-2 \alpha) r} .
$$

7. Two-point distortion theorems. In this section, we present twopoint distortion theorems for functions in $K_{h}^{1}$. The method is very similar to that employed in [6] in which two-point distortion theorems for bounded univalent functions were established. If a proof is similar to that for bounded univalent functions, we do not hesitate to omit some of the details. Note that a different normalization of the hyperbolic metric was employed in [6], so some formulas here are slightly different from the corresponding formulas in $[6]$.

Assume $\gamma: z=z(s),-L \leq s \leq L$, is a smooth path in $\mathbb{D}$ parametrized by hyperbolic arclength. For any real number $p \neq 0$, set

$$
v(s)=\left(\frac{\left|D_{h 1} f(z(s))\right|}{1-\left|D_{h 1} f(z(s))\right|}\right)^{p} .
$$

Then (see [6])

$$
v^{\prime}(s)=\frac{p v(s)}{2\left(1-\left|D_{h 1} f(z(s))\right|\right)} \operatorname{Re}\left\{e^{i \theta(s)} Q_{f}(z(s))\right\},
$$




$$
\begin{aligned}
& \frac{4\left(1-\left|D_{h 1} f(z(s))\right|\right)}{p v(s)} v^{\prime \prime}(s) \\
& =\operatorname{Re}\left\{e^{i 2 \theta(s)}\left(\left(1-|z(s)|^{2}\right)^{2} S_{f}(z(s))+\frac{1}{2} Q_{f}^{2}(z(s))\right)\right\} \\
& \quad+\frac{p+\left|D_{h 1} f(z(s))\right|}{1-\left|D_{h 1} f(z(s))\right|} \operatorname{Re}^{2}\left\{e^{i \theta(s)} Q_{f}(z(s))\right\} \\
& \quad-2 \kappa_{\mathbb{D}}(z(s), \gamma) \operatorname{Im}\left\{e^{i \theta(s)} Q_{f}(z(s))\right\}-2+2\left|D_{h 1} f(z(s))\right|^{2}
\end{aligned}
$$

and so

$$
\begin{aligned}
& \frac{4\left(1-\left|D_{h 1} f(z(s))\right|\right)}{p v(s)} v^{\prime \prime}(s) \\
& =\operatorname{Re}\left\{e^{i 2 \theta(s)}\left(\left(1-|z(s)|^{2}\right)^{2} S_{f}(z(s))+\frac{1}{2} Q_{f}^{2}(z(s))\right)\right\} \\
& \quad+\frac{p+\left|D_{h 1} f(z(s))\right|}{1-\left|D_{h 1} f(z(s))\right|} \operatorname{Re}^{2}\left\{e^{i \theta(s)} Q_{f}(z(s))\right\}+\operatorname{Im}^{2}\left\{e^{i \theta(s)} Q_{f}(z(s))\right\} \\
& \quad-2 \kappa_{\mathbb{D}}(f(z(s)), f \circ \gamma)\left|D_{h 1} f(z(s))\right| \operatorname{Im}\left\{e^{i \theta(s)} Q_{f}(z(s))\right\} \\
& \quad-2+2\left|D_{h 1} f(z(s))\right|^{2} .
\end{aligned}
$$

Here $\kappa_{\mathbb{D}}(z(s), \gamma)$ denotes the hyperbolic curvature of $\gamma$ at $z(s)$, with a similar interpretation of the term $\kappa_{\mathbb{D}}(f(z(s)), f \circ \gamma)$.

The following integral inequalities were established in [6].

Proposition 7.1. Suppose $v \in C^{2}[-L, L], v>0, k>0, p \geq 1,\left|v^{\prime}\right| \leq$ $k p v$ and $v^{\prime \prime} \leq k^{2} p^{2} v$. Then the following inequalities hold:

$$
2 L+\frac{1}{k} \log \frac{\left(1+e^{-2 k p L}\right)^{1 / p}+[v(L)+v(-L)]^{1 / p}}{\left(1+e^{2 k p L}\right)^{1 / p}+[v(L)+v(-L)]^{1 / p}} \leq \int_{-L}^{L} \frac{v(s)^{1 / p}}{1+v(s)^{1 / p}} d s
$$

and equality holds if and only if $v(s)=A e^{ \pm k p s}, A>0$; and

$$
\begin{aligned}
& \int_{-L}^{L} \frac{1}{1+v(s)^{1 / p}} d s \\
& \quad \leq 2 L+\frac{1}{k} \log \frac{\left(1+e^{2 k p L}\right)^{-1 / p}+[v(L)+v(-L)]^{-1 / p}}{\left(1+e^{-2 k p L}\right)^{-1 / p}+[v(L)+v(-L)]^{-1 / p}}
\end{aligned}
$$

and equality holds if and only if $v(s)=A e^{ \pm k p s}, A>0$.

Theorem 7.2. Suppose $f \in K_{h}^{1}$.

(a) For $a, b \in \mathbb{D}$ and $p \geq 1$,

$$
d_{\mathbb{D}}(a, b)+E_{f}(a, b ; p) \leq d_{\mathbb{D}}(f(a), f(b)),
$$


where

$E_{f}(a, b ; p)$

$$
=\log \frac{\left(1+e^{-p d_{\mathbb{D}}(a, b)}\right)^{1 / p}+\left[\left(\frac{\left|D_{h 1} f(a)\right|}{1-\left|D_{h 1} f(a)\right|}\right)^{p}+\left(\frac{\left|D_{h 1} f(b)\right|}{1-\left|D_{h 1} f(b)\right|}\right)^{p}\right]^{1 / p}}{\left(1+e^{p d_{\mathbb{D}}(a, b)}\right)^{1 / p}+\left[\left(\frac{\left|D_{h 1} f(a)\right|}{1-\left|D_{h 1} f(a)\right|}\right)^{p}+\left(\frac{\left|D_{h 1} f(b)\right|}{1-\left|D_{h 1} f(b)\right|}\right)^{p}\right]^{1 / p}} .
$$

(b) For $a, b \in \mathbb{D}$ and $p \geq 1.5$,

$$
d_{\mathbb{D}}(f(a), f(b)) \leq d_{\mathbb{D}}(a, b)+E_{f}(a, b ;-p) .
$$

These inequalities are identities on $(-1,1)$ for $k_{\alpha}$.

Proof. (a) Fix $a, b \in \mathbb{D}$. Since $f \in K_{h}^{1}$, the hyperbolic geodesic $\Gamma$ connecting $f(a)$ and $f(b)$ lies in $f(\mathbb{D})$. Set $\gamma=f^{-1} \circ \Gamma$. Then $\gamma$ is a smooth arc in $\mathbb{D}$ joining $a$ and $b$. Suppose $\gamma: z=z(s),-L \leq s \leq L$, is a hyperbolic arclength parametrization of $\gamma$. Then $2 L \geq d_{\mathbb{D}}(a, b)$ and $\kappa_{\mathbb{D}}(f(z(s)), f \circ \gamma)=0$. For $p \geq 1$, we consider the function $v(s)$ defined in (7.1). Then (7.2) and (2.2) give $\left|v^{\prime}(s)\right| \leq p v(s)$. By using (7.4) and $\operatorname{Re}^{2}\{z\}=\frac{1}{2} \operatorname{Re}\left\{z^{2}\right\}+\frac{1}{2}|z|^{2}$, we have

$$
\begin{aligned}
& \frac{4\left(1-\left|D_{h 1} f(z(s))\right|\right)}{p v(s)} v^{\prime \prime}(s) \\
& =\operatorname{Re}\left\{e^{i 2 \theta(s)}\left(\left(1-|z(s)|^{2}\right)^{2} S_{f}(z(s))+\frac{1}{2} Q_{f}^{2}(z(s))\right)\right\} \\
& \quad+\frac{p+\left|D_{h 1} f(z(s))\right|}{1-\left|D_{h 1} f(z(s))\right|} \operatorname{Re}^{2}\left\{e^{i \theta(s)} Q_{f}(z(s))\right\}+\operatorname{Im}^{2}\left\{e^{i \theta(s)} Q_{f}(z(s))\right\} \\
& \quad-2+2\left|D_{h 1} f(z(s))\right|^{2} .
\end{aligned}
$$

Wirths [16] proved

$$
\left(1-|z|^{2}\right)^{2}\left|S_{f}(z)\right| \leq 2\left(1-\left|D_{h 1} f(z)\right|\right)-\frac{\left|Q_{f}(z)\right|^{2}}{2\left(1-\left|D_{h 1} f(z)\right|\right)} .
$$

This is an identity on $(-1,1)$ for $k_{\alpha}$. From (7.7) and (2.2), we obtain

$$
\begin{aligned}
& \frac{4\left(1-\left|D_{h 1} f(z(s))\right|\right)}{p v(s)} v^{\prime \prime}(s) \\
& \quad \leq \frac{2 p+\left|D_{h 1} f(z(s))\right|}{2\left(1-\left|D_{h 1} f(z(s))\right|\right)}\left|Q_{f}(z(s))\right|^{2}+2\left|D_{h 1} f(z(s))\right|^{2}-2\left|D_{h 1} f(z(s))\right| \\
& \leq 4 p\left(1-\left|D_{h 1} f(z(s))\right|\right) .
\end{aligned}
$$

Thus, $v(s)$ satisfies the hypotheses of Proposition 7.1 for $k=1$ and $p \geq 1$. Since

$$
d_{\mathbb{D}}(f(a), f(b))=\int_{-L}^{L} \frac{v(s)^{1 / p}}{1+v(s)^{1 / p}} d s
$$

$2 L \geq d_{\mathbb{D}}(a, b)$ and the left-hand side of (7.5) is an increasing function of $L$, 
(7.5) implies

$$
\begin{aligned}
d_{\mathbb{D}}(f(a), f(b)) & \geq 2 L+\log \frac{\left(1+e^{-2 p L}\right)^{1 / p}+[v(L)+v(-L)]^{1 / p}}{\left(1+e^{2 p L}\right)^{1 / p}+[v(L)+v(-L)]^{1 / p}} \\
& \geq d_{\mathbb{D}}(a, b)+\log \frac{\left(1+e^{-p d_{\mathbb{D}}(a, b)}\right)^{1 / p}+[v(L)+v(-L)]^{1 / p}}{\left(1+e^{p d_{\mathbb{D}}(a, b)}\right)^{1 / p}+[v(L)+v(-L)]^{1 / p}} .
\end{aligned}
$$

This is equivalent to the inequality in (a).

(b) Fix $a, b \in \mathbb{D}$. Let $\gamma$ be the hyperbolic geodesic arc between $a$ and $b$, and $\gamma: z=z(s),-L \leq s \leq L$, the hyperbolic arclength parametrization of $\gamma$. In this situation, $d_{\mathbb{D}}(a, b)=2 L$ and $\kappa_{\mathbb{D}}(z(s), \gamma)=0$. This time we take

$$
v(s)=\left(\frac{\left|D_{h 1} f(z(s))\right|}{1-\left|D_{h 1} f(z(s))\right|}\right)^{-p},
$$

where $p>0$. From (7.2) and (2.2), we have $\left|v^{\prime}(s)\right| \leq p v(s)$. It follows from (7.3) and $\operatorname{Re}^{2}\{z\}=\frac{1}{2} \operatorname{Re}\left\{z^{2}\right\}+\frac{1}{2}|z|^{2}$ that

$$
\begin{aligned}
\left.\frac{4(1-}{p v(s)}\left|D_{h 1} f(z(s))\right|\right) & v^{\prime \prime}(s) \\
= & -\operatorname{Re}\left\{e^{i 2 \theta(s)}\left(\left(1-|z(s)|^{2}\right)^{2} S_{f}(z(s))+\frac{1}{2} Q_{f}^{2}(z(s))\right)\right\} \\
& +\frac{p-\left|D_{h 1} f(z(s))\right|}{1-\left|D_{h 1} f(z(s))\right|} \operatorname{Re}^{2}\left\{e^{i \theta(s)} Q_{f}(z(s))\right\}+2-2\left|D_{h 1} f(z(s))\right|^{2} \\
\leq & \left(1-|z(s)|^{2}\right)^{2}\left|S_{f}(z(s))\right|+\frac{2 p-1-\left|D_{h 1} f(z(s))\right|}{2\left(1-\left|D_{h 1} f(z(s))\right|\right)}\left|Q_{f}(z(s))\right|^{2} \\
& +2-2\left|D_{h 1} f(z(s))\right|^{2} .
\end{aligned}
$$

By using (7.7) and (2.2) and assuming $p \geq 1.5$, we obtain

$$
\frac{4\left(1-\left|D_{h 1} f(z(s))\right|\right)}{p v(s)} v^{\prime \prime}(s) \leq 4 p\left(1-\left|D_{h 1} f(z(s))\right|\right) .
$$

Hence, $v$ satisfies the conditions of Proposition 7.1 for $k=1$ and $p \geq 1.5$. In this case,

$$
d_{\mathbb{D}}(f(a), f(b)) \leq \int_{f \circ \gamma} \lambda_{\mathbb{D}}(w)|d w|=\int_{-L}^{L} \frac{1}{1+v(s)^{1 / p}} d s .
$$

By applying $(7.6)$ and $d_{\mathbb{D}}(a, b)=2 L$, we find

$$
\begin{aligned}
d_{\mathbb{D}}(f(a), f(b)) & \leq 2 L+\log \frac{\left(1+e^{2 p L}\right)^{-1 / p}+[v(L)+v(-L)]^{-1 / p}}{\left(1+e^{-2 p L}\right)^{-1 / p}+[v(L)+v(-L)]^{-1 / p}} \\
& =d_{\mathbb{D}}(a, b)+\log \frac{\left(1+e^{p d_{\mathbb{D}}(a, b)}\right)^{-1 / p}+[v(L)+v(-L)]^{-1 / p}}{\left(1+e^{-p d_{\mathbb{D}}(a, b)}\right)^{-1 / p}+[v(L)+v(-L)]^{-1 / p}} .
\end{aligned}
$$

This is equivalent to the inequality in (b). 
We conjecture that the upper bound in Theorem 7.2 remains valid for $p \geq 1$. The lower bound in part (a) is a decreasing function of $p$ while the upper bound in (b) is an increasing function of $p$. The weakest inequalities in Theorem 7.2 are as follows when $p=\infty$.

Corollary 7.3. Suppose $f \in K_{h}^{1}$. Then for $a, b \in \mathbb{D}$,

$$
\begin{aligned}
& d_{\mathbb{D}}(a, b)+\log \frac{1+\max \left\{\frac{\left|D_{h 1} f(a)\right|}{1-\left|D_{h 1} f(a)\right|}, \frac{\left|D_{h 1} f(b)\right|}{1-\left|D_{h 1} f(b)\right|}\right\}}{e^{d_{\mathbb{D}}(a, b)}+\max \left\{\frac{\left|D_{h 1} f(a)\right|}{1-\left|D_{h 1} f(a)\right|}, \frac{\left|D_{h 1} f(b)\right|}{1-\left|D_{h 1} f(b)\right|}\right\}} \\
& \leq d_{\mathbb{D}}(f(a), f(b)) \leq d_{\mathbb{D}}(a, b)+\log \frac{e^{-d_{\mathbb{D}}(a, b)}+\min \left\{\frac{\left|D_{h 1} f(a)\right|}{1-\left|D_{h 1} f(a)\right|}, \frac{\left|D_{h 1} f(b)\right|}{1-\left|D_{h 1} f(b)\right|}\right\}}{1+\min \left\{\frac{\left|D_{h 1} f(a)\right|}{1-\left|D_{h 1} f(a)\right|}, \frac{\left|D_{h 1} f(b)\right|}{1-\left|D_{h 1} f(b)\right|}\right\}} .
\end{aligned}
$$

This corollary can be regarded as a refinement for functions in $K_{h}^{1}$ of the Schwarz-Pick Lemma. It is the invariant version of the growth theorem (2.3) for $K_{h}^{1}$. If we choose $a=0, b=z$ in Corollary 7.3, we obtain

$$
\log \frac{1}{1-\alpha+\alpha e^{-d_{\mathbb{D}}(0, z)}} \leq d_{\mathbb{D}}(0, f(z)) \leq \log \left(1-\alpha+\alpha e^{d_{\mathbb{D}}(0, z)}\right),
$$

or

$$
\frac{1}{1-\alpha+\alpha \frac{1-|z|}{1+|z|}} \leq \frac{1+|f(z)|}{1-|f(z)|} \leq 1-\alpha+\alpha \frac{1+|z|}{1-|z|}
$$

as $\log \frac{1+x}{1+x e^{-d_{\mathbb{D}}(0, z)}}$ and $\log \frac{1+x e^{d_{\mathbb{D}}(0, z)}}{1+x}$ are increasing functions of $x$. This is equivalent to $(2.3)$.

Remarks. The weakest inequalities in Theorem 7.2 imply $f \in K_{h}^{1}$. Precisely, if $f \in \mathcal{B}$ satisfies either of the inequalities in Corollary 7.3, then $f \in K_{h}^{1}$. To see this, it suffices to show that each of the inequalities in Corollary 7.3 implies (2.2). Because the inequalities in Corollary 7.4 are invariant when we replace $f$ by $S \circ f \circ T$, where $S, T$ are conformal automorphisms of $\mathbb{D}$, we may assume $f(0)=0$ and it is enough to show $\left|f^{\prime \prime}(0) / f^{\prime}(0)\right| \leq 2\left(1-\left|f^{\prime}(0)\right|\right)$. Inequalities (2.3) implies

$$
\frac{\left|f^{\prime}(0)\right||z|}{1+\left(1-\left|f^{\prime}(0)\right|\right)|z|} \leq|f(z)| \leq \frac{\left|f^{\prime}(0)\right||z|}{1-\left(1-\left|f^{\prime}(0)\right|\right)|z|}
$$

or

$$
\begin{aligned}
|z|\left(1-\left(1-\left|f^{\prime}(0)\right|\right)|z|+o(|z|)\right) & \leq\left|f(z) / f^{\prime}(0)\right| \\
& \leq|z|\left(1+\left(1-\left|f^{\prime}(0)\right|\right)|z|+o(|z|)\right) .
\end{aligned}
$$

Since

$$
\left|f(z) / f^{\prime}(0)\right|=|z|\left(1+\operatorname{Re}\left\{z f^{\prime \prime}(0) /\left(2 f^{\prime}(0)\right)\right\}+o(|z|)\right),
$$

we have

$$
-\left(1-\left|f^{\prime}(0)\right|\right)|z|+o(|z|) \leq \operatorname{Re}\left\{z f^{\prime \prime}(0) /\left(2 f^{\prime}(0)\right)\right\} \leq\left(1-\left|f^{\prime}(0)\right|\right)|z|+o(|z|) .
$$


For $z=r e^{i \theta}$ we obtain

$$
-\left(1-\left|f^{\prime}(0)\right|\right)+o(1) \leq \operatorname{Re}\left\{e^{i \theta} f^{\prime \prime}(0) /\left(2 f^{\prime}(0)\right)\right\} \leq 1-\left|f^{\prime}(0)\right|+o(1) .
$$

Since $\theta$ is arbitrary, either inequality implies $\left|f^{\prime \prime}(0) / f^{\prime}(0)\right| \leq 2\left(1-\left|f^{\prime}(0)\right|\right)$.

As in the proof of Corollary 4 in [6], we obtain the following comparison theorem between hyperbolic geometry on hyperbolically 1-convex region $\Omega$ and hyperbolic geometry on $\mathbb{D}$ by using the lower bound in Corollary 7.3.

Corollary 7.4. Suppose $\Omega \subset \mathbb{D}$ is hyperbolically 1 -convex, $\Omega \neq \mathbb{D}$. Then for $w \in \Omega$,

$$
\lambda_{\Omega}(w) \geq \frac{\lambda_{\mathbb{D}}(w)}{1-\exp \left(-\varepsilon_{\Omega}(w)\right)},
$$

where $\varepsilon_{\Omega}(w)=\inf \left\{d_{\mathbb{D}}(w, \omega): \omega \in \partial \Omega\right\}$.

REMARKS. Corollary 7.4 is an invariant version of a covering theorem for $K_{h}^{1}$. If $f \in K_{h}^{1}(\alpha)$, then (see [9]) $f(\mathbb{D}) \supset\{w:|w|<\alpha /(2-\alpha)\}$. Set $\Omega_{\alpha}=k_{\alpha}(\mathbb{D})$. Then $\lambda_{\Omega_{\alpha}}(0)=2 / \alpha$ and $\varepsilon_{\Omega_{\alpha}}(0)=d_{\mathbb{D}}(0,-\alpha /(2-\alpha))=$ $-\log (1-\alpha)$. This implies

$$
\frac{\lambda_{\mathbb{D}}(0)}{1-\exp \left(-\varepsilon_{\Omega_{\alpha}}(0)\right)}=\frac{2}{\alpha}=\lambda_{\Omega_{\alpha}}(0) .
$$

Thus the inequality in Corollary 7.4 is best possible.

\section{References}

[1] M. Chuaqui and B. Osgood, Sharp distortion theorems associated with the Schwarzian derivative, J. London Math. Soc. 48 (1993), 289-298.

[2] R. Fournier, J. Ma and S. Ruscheweyh, Convex univalent functions and omitted values, in: Approximation Theory, Monogr. Textbooks Pure Appl. Math. 212, Dekker, 1998, 225-241.

[3] W. Ma and D. Minda, A unified treatment of some special classes of univalent functions, in: Proc. Conference on Complex Analysis (Tianjin, 1992), Conf. Proc. Lecture Notes Anal. I, Internat. Press, Cambridge, MA, 1994, 157-169.

[4] - - - Hyperbolic linear invariance and hyperbolic $k$-convexity, J. Austral. Math. Soc. Ser. A 58 (1995), 73-93.

[5] —, - Hyperbolically convex functions, Ann. Polon. Math. 60 (1994), 81-100.

[6] -, - Two-point distortion theorems for bounded univalent functions, Ann. Acad. Sci. Fenn. Math. 22 (1997), 425-444.

[7] —, - Hyperbolically convex functions II, Ann. Polon. Math. 71 (1999), 273-285.

[8] W. Ma, D. Minda and D. Mejia, Distortion theorems for hyperbolically and spherically $k$-convex functions, in: Proc. Internat. Conf. on New Trends in Geometric Function Theory and Appl., R. Parvatham and S. Ponnusamy (eds.), World Sci., Singapore, 1991, 46-54.

[9] D. Mejia and D. Minda, Hyperbolic geometry in hyperbolically k-convex regions, Rev. Colombiana Mat. 25 (1991), 123-142. 
[10] D. Mejia and Ch. Pommerenke, On spherically convex functions, Michigan Math. J. 47 (2000), 163-172.

[11] —, - On hyperbolically convex functions, J. Geom. Anal. 10 (2000), 365-378.

[12] S. Ruscheweyh, Convolutions in Geometric Function Theory, Presses Univ. de Montréal, Montréal, 1982.

[13] T. Sheil-Small, On convex univalent functions, J. London Math. Soc. 1 (1969), 483-492.

[14] T. J. Suffridge, Some remarks on convex maps of the unit disk, Duke Math. J. 37 (1970), 775-777.

[15] S. Y. Trimble, A coefficient inequality for convex univalent functions, Proc. Amer. Math. Soc. 48 (1975), 266-267.

[16] K.-J. Wirths, Verallgemeinerungen eines Maximumprinzips, Bonner Math. Schriften 51 (1971), 61 pp.

School of Integrated Studies

Pennsylvania College of Technology

Williamsport, PA 17701, U.S.A.

E-mail:wma@pct.edu

Departamento de Matemáticas

Universidad Nacional de Colombia

A.A. 3840, Medellín, Colombia

E-mail: dmejia@perseus.unalmed.edu.co
Department of Mathematical Sciences

University of Cincinnati Cincinnati, OH 45221-0025, U.S.A. E-mail: David.Minda@math.uc.edu

Reçu par la Rédaction le 14.8.2004

Révisé le 30.11.2004 